Références bibliographiques du dossier « la fragmentation des systèmes scolaires nationaux »

Bernadette Plumelle

\title{
OpenEdition
}

1 Journals

Édition électronique

URL : https://journals.openedition.org/ries/6087

DOI : $10.4000 /$ ries. 6087

ISSN : 2261-4265

Éditeur

France Education international

Édition imprimée

Date de publication : 1 décembre 2017

Pagination : 147-158

ISBN : 978-2-85420-616-6

ISSN : 1254-4590

Référence électronique

Bernadette Plumelle, «Références bibliographiques du dossier « la fragmentation des systèmes scolaires nationaux » », Revue internationale d'éducation de Sèvres [En ligne], 76 | décembre 2017, mis en ligne le 01 décembre 2017, consulté le 25 juin 2021. URL : http://journals.openedition.org/ries/6087 ; DOl : https://doi.org/10.4000/ries.6087 


\section{Références bibliographiques du dossier " la fragmentation des systèmes scolaires nationaux $"$}

\section{Bernadette Plumelle \\ CIEP}

Processus de mondialisation, mouvement de repli communautaire, développement d'une offre d'établissements privés, aspiration des parents au libre choix de l'école, ségrégation scolaire, multiplication des réseaux d'éducation, offre alternative d'enseignement... Couvrir l'ensemble des problématiques portées par ce dossier est particulièrement difficile pour cette sélection bibliographique qui ne prétend pas couvrir toutes les questions de manière approfondie.

On a choisi de sélectionner des études récentes, principalement de langue française, en les organisant selon plusieurs points d'entrée. La première partie, à valeur introductive, propose des références dont le dénominateur commun est celui des profonds changements en cours de la forme scolaire. Les parties suivantes ont pour sujet : le processus de mondialisation et la diversification des systèmes éducatifs, les marchés scolaires et le développement de l'enseignement privé, la concurrence entre les établissements scolaires, la ségrégation scolaire et le communautarisme. Les deux dernières parties, plus succinctes, visent à donner un aperçu de la variété des réseaux éducatifs internationaux et des écoles alternatives.

Les références proposées complètent celles fournies par les auteurs des articles du dossier et, à quelques exceptions près, ne sont pas reprises ici. Les résumés sont, pour l'essentiel, ceux des éditeurs et des revues.

Bibliographie arrêtée le 13 novembre 2017.

\section{LES MUTATIONS DE LA FORME SCOLAIRE}

Barrère Anne (coord.), "Les établissements à l'heure des "dispositifs" ", Carrefours de l'éducation, 2013, $n^{\circ} 36,248 p$.

Le projet de ce dossier est de s'interroger sur la réalité couverte par le terme de "dispositif », un mot de plus en plus employé dans le champ de l'éducation, sans définition précise, à propos de multiples actions éducatives en partie périphériques de la classe traditionnelle (soutien, inclusion, lutte contre la violence, décrochage, etc.). Ces dispositifs apparaissent comme intermédiaires entre remises en question de la forme scolaire et maintien de celle-ci en l'état; ils en organisent des inflexions contextualisées répondant à une volonté de faire face à des problèmes jugés irrésolus dans le cadre du fonctionnement ordinaire de l'école.

DELVAUX Bernard, ALBARELLO Luc, BOUHON Mathieu (éd.), Réfléchir l'école de demain, De Boeck/Bruxelles, 2015, 183 p., bibliogr.

De nos jours, l'école est souvent décriée et sommée de s'adapter aux évolutions sociétales. Mais où mènent les multiples réformes et innovations mises en place en réponse à ces pressions ? $\mathrm{Ne}$ contribuent-elles pas à dissoudre la forme particulière d'éducation qu'incarnait l'école ? $\mathrm{Ne}$ 
l'éloignent-elles pas de ses finalités émancipatrices? Ne fragilisent-elles pas l'institution scolaire, au point de la fragmenter en organisations éducatives de plus en plus différenciées ? Les auteurs replacent l'école dans l'histoire et la société. Ils cherchent à prendre du recul et à poser des questions essentielles, trop peu débattues. Leur objectif est d'identifier certains enjeux fondamentaux, masqués par l'amoncellement d'enjeux mineurs, et d'imaginer de nouvelles perspectives pour une éducation émancipatrice.

\section{DURPAIRE François, MABILON-BONFILS Béatrice, La fin de l'école : l'ère du savoir-relation, Presses universitaires de France/Paris, 2014, 273 p.}

Le tournant global et la révolution numérique interrogent la raison d'être d'une école en crise de sens. Ces mutations questionnent autant la production des savoirs que l'éducation au monde dispensée par l'École. La notion même de savoir est remise en cause par la culture numérique. Les robots vont-ils remplacer les professeurs? Le lieu scolaire doit-il disparaître ? Et si la fin de l'École était une opportunité pour créer de nouveaux espaces, de nouvelles relations enseignants/enseignés, pour réconcilier les partenaires, enseigner autrement et recréer le bonheur d'apprendre et donc refonder notre rapport au savoir?

GEAY Bertrand, MILLET Mathias, CROIZET Jean-Claude, et al., "Les temps des apprentissages : quelles continuités éducatives ? ", Diversités $n^{\circ} 183,2016,167$ p., bibliogr.

À l'heure où les modalités d'apprentissage se diversifient considérablement, où les occasions d'apprendre sont de plus en plus nombreuses, l'idée d'une éducation globale tend à s'affirmer parmi les acteurs de l'éducation, avec l'ambition de mettre en continuité et en cohérence les rôles respectifs de l'école, des familles, des acteurs sociaux et/ou des territoires. Ce numéro explore la question des continuités/discontinuités éducatives et de la cohérence des parcours d'apprentissage, ainsi que celle des articulations entre des espaces-temps éducatifs multiples.

\section{MAULINI Olivier coord., PROGIN Laetitia coord., Des établissements scolaires autonomes? Entre inventivité des acteurs et éclatement du système, ESF/Paris, 2016, 192 p., bibliogr. (Pédagogies)}

Faut-il rendre les établissements scolaires autonomes ? La décentralisation serait-elle le remède idéal ou la porte ouverte à la compétition généralisée ? L'ouvrage dresse un bilan prospectif et comparatif de la situation en France, en Belgique, en Suisse et au Québec, en confrontant les histoires, les systèmes d'organisation et les réformes. La première partie montre comment la question de l'autonomie des établissements est revisitée par des politiques éducatives en quête d'efficacité, de traçabilité et de légitimité. La deuxième partie s'approche du terrain pour observer qui décide (et comment), lorsqu'un établissement gagne en autonomie. La troisième partie s'intéresse aux acteurs.

\footnotetext{
MESKEL-CRESTA M., NORDMANN J.-F., BONGRAND Ph., et al., École et mutation : reconfigurations, résistances, émergences, De Boeck/Louvain-laNeuve, 2014, 407 p.

L'ouvrage cherche à identifier les mutations subies ou produites par l'École en multipliant et croisant les angles, les domaines et les échelles d'analyse, du plus large (reconfigurations sociétales) au plus fin (expériences et pratiques des acteurs de terrain) et en considérant en retour les effets des pratiques et de leurs transformations aux niveau pédagogique, didactique, disciplinaire et symbolique. Les contributeurs, spécialistes en sciences sociales et humaines, donnent à voir un espace de réflexion ouvert et interdisciplinaire et entreprennent, au moyen de ce "prisme » de la mutation, de produire une vision renouvelée et intégrée des bouleversements actuels de l'École et de ses reconfigurations.
} 
TARDIF Maurice, MARCEL Jean-François, PERISSET Danièle (sous la direction de), L'organisation du travail des acteurs scolaires : points de repères sur les évolutions au début du XXI siècle, PUL : Presse de l'université de Laval/Laval, 2017, $178 \mathrm{p}$.

Décentralisation des systèmes scolaires, concurrence entre les établissements, nouveaux modes de régulation de l'éducation et nouvelles politiques éducatives, pression pour le travail collectif, professionnalisation de l'enseignement, programmes scolaires basés sur des compétences, ouverture de l'école aux parents et aux communautés locales, introduction des TIC : l'organisation scolaire connaît, depuis une trentaine d'années, de nombreux changements. L'ouvrage décrit, analyse et conceptualise la situation de l'école à la lumière des transformations de l'organisation du travail des acteurs scolaires en France, au Québec et en Suisse.

VINCENT Guy (sous la direction de), L'éducation prisonnière de la forme scolaire? Scolarisation et socialisation dans les sociétés industrielles, Presses universitaires de Lyon/Lyon, 1994, 227 p.

Qu'entend-t-on par "forme scolaire » ? C'est ce concept que veut expliquer l'auteur dans son introduction. Puis, dans la première partie, il présente une théorie de la forme scolaire, élaborée par des chercheurs de l'Université Lumière à Lyon ; dans la deuxième partie, ces derniers mettent en ouvre par leurs contributions cette théorie.

\section{MONDIALISATION / DIVERSIFICATION DES SYSTÈMES SCOLAIRES}

CHARLOT Bernard, "Convergence internationale et diversification interne des modèles scolaires", Revue internationale d'éducation Sèvres, décembre 2009, $n^{\circ}$ 52, p. 123-127 [en ligne]

Le colloque de la Revue internationale d'éducation de Sèvres "Un seul monde, une seule école? » (mars 2009) a tout à la fois constaté la diversité des écoles et montré que, dans de nombreux pays, elles affrontent des problèmes et des défis qui sont des effets de la globalisation. La diversité des systèmes éducatifs découle de celle des valeurs sociales dans lesquelles ils s'enracinent. Les résultats des évaluations internationales comme PISA montrent que des systèmes éducatifs très différents répondent avec succès aux exigences de la globalisation. Toutefois, les pressions de la globalisation induisent une différenciation interne des systèmes scolaires, entre un réseau d'éducation défini par des standards globaux et, pour les plus pauvres, des écoles fondées sur des valeurs nationales. [https://ries.revues.org/785]

EGIDO GALVEZ Inmaculada, "Influencias transnacionales en las políticas educativas : los modelos de evaluación del sistema escolar en México y España ", Revista Iberoamericana de Educación, juillet 2016, vol. 71, $n^{\circ}$ 2, p. 191-210. [en ligne]

Il existe actuellement un débat sur les conséquences de la mondialisation dans le domaine de l'éducation. Certains experts défendent l'existence d'une harmonisation progressive des politiques d'éducation à l'échelle mondiale, tandis que d'autres considèrent que le poids des États nationaux reste décisif dans l'élaboration des systèmes éducatifs. Cet article analyse et compare les modèles d'évaluation de deux systèmes scolaires aux caractéristiques très différentes, le Mexique et l'Espagne. Les résultats indiquent une convergence entre ces deux pays, qui ont adopté des modèles d'évaluation du système scolaire pratiquement identiques.

[http://g00.g/03PS6n] 
LANGE Marie-France, HENAFF Nolwen (sous la direction de), "Internationalisation et transformation des systèmes éducatifs au Sud", Revue Tiers Monde, septembre 2015, $n^{\circ} 223,233 p$.

Les différents niveaux et ordres d'enseignement (public, privé laïc ou confessionnel) sont touchés par les phénomènes d'internationalisation. Ce numéro réunit des articles qui portent sur plusieurs régions du Sud (Afrique sub-saharienne, Amérique du Sud, Maghreb) et en particulier sur le Bénin, la Bolivie, la Côte d'Ivoire, Djibouti, la République démocratique du Congo, le Sénégal. Les contributeurs concentrent leurs études sur les modalités de l'internationalisation ainsi que sur la manière dont s'élaborent les politiques publiques nationales d'éducation et se construisent les stratégies des différents acteurs dans le processus de mondialisation.

LEWANDOWSKI Sophie, "Politiques de lutte contre la pauvreté et inégalités scolaires à Dakar : vers un éclatement des normes éducatives? ", Autrepart, $n^{\circ}$ 59, mars 2011, p. 37-56

Au Sénégal, les politiques de lutte contre la pauvreté et l'Éducation pour tous (EPT) ont repositionné l'État dans la question éducative. Mais la mise en place d'un partenariat entre État, bailleurs et société civile n'a permis ni un pilotage national de l'école ni une démocratisation des décisions éducatives. De plus, les politiques de l'EPT ont favorisé une perte de crédibilité de l'école publique en faveur des écoles privées confessionnelles, ce qui entraîne de nouvelles formes d'inégalités scolaires et sociales: les familles aisées développent des stratégies de surenchère éducative pour améliorer la performance scolaire de leurs enfants, alors que les moins aisées optent pour un contournement de l'impératif scolaire. [https://goo.gl/H2wnhP]

MALET Régis, MANGEZ Eric (coord.), "Éducation et mondialisation : dossier », Spirale : revue de recherches en éducation, 2013, $n^{\circ} 51$, p. 3-180

La période contemporaine est marquée par un vaste mouvement de recomposition des mondes éducatifs et scolaires. Sous l'effet de pressions tant exogènes qu'endogènes, on observe une intensification des relations d'interdépendance entre les systèmes d'éducation et de formation, qui s'exprime notamment en termes d'émergence de nouveaux acteurs dans l'espace public, de contractualisation des objectifs et de pilotage par la performance, de réformes organisationnelles et curriculaires convergentes, d'imputabilité des institutions et des professionnels de l'éducation. Ce numéro rassemble des recherches qui mettent au jour les diversités nationales, régionales et locales qui s'expriment en creux d'un mouvement global de convergence formelle des systèmes scolaires, en pensant à la fois ce mouvement sur le temps long, et à partir d'études empiriques locales, qui éclairent les effets de la mondialisation dans des contextes éducatifs et de travail.

PROST Yannick, "Les politiques publiques de l'éducation : la grande convergence mondiale?", Après-demain, 2016/4, n 40, p. 13-15

La plupart des gouvernements sont conscients de l'enjeu de l'amélioration du niveau d'éducation, soit pour des questions d'équité, soit plus sûrement pour des raisons d'amélioration de la qualité de la main-d'œuvre et de la compétitivité de l'économie nationale. Les organisations internationales (OCDE, Banque mondiale, Unesco) ont diffusé des préconisations qui les complètent mais peuvent s'avérer en contradiction avec celles du new public management. Dans les pays développés, public et privé cohabitent habituellement, dans des proportions variables. Si les préconisations en matière de réforme n'ont pas insisté sur la privatisation du système scolaire, elles ont en revanche visé à la décentralisation, à l'autonomie des écoles ou au choix de l'établissement par les parents. La tendance plus générale au creusement des inégalités sociales n'est pas étrangère à la crispation d'une partie de l'opinion publique - la plus favorisée - sur le choix de l'école, la décentralisation ou la privatisation, afin de renforcer par la ségrégation scolaire l'avantage acquis par la reproduction du capital social. 
TEODORO Antonio, PANAIT Oana Marina (coord.), "Résistances au nouvel ordre éducatif mondial : dossier ", Éducation et sociétés, 2017, n 39, 2017/1, p. 5-135, bibliogr.

Depuis les années 1990, l'échelon national a cessé d'être celui qui rend intelligibles les politiques d'enseignement. Le processus de standardisation des références des politiques publiques demande de prendre en compte l'international et l'infranational et d'analyser les manières dont des acteurs établissent des connexions stratégiques entre ces échelons. La mise en place du nouvel ordre mondial puisant ses repères en Occident, ce dossier fait le point sur la dynamique de recherche sur les résistances qui naissent, au Nord et au Sud, face aux normes issues de la globalisation.

\title{
LES MARCHÉS SCOLAIRES
}

\author{
DRAELANTS Hugues, DUMAY Xavier (sous la direction de), Les écoles et leur \\ réputation: l'identité des établissements en contexte de marché, De Boeck \\ Supérieur/Louvain-la-Neuve, 2016, 271 p.
}

Les marchés scolaires sont aujourd'hui une réalité dans de nombreux systèmes éducatifs. Cet ouvrage se centre sur les effets pratiques et symboliques des marchés pour le fonctionnement et l'identité des écoles. Il souligne notamment combien la réputation prend de l'importance dans ce contexte. La maîtrise des images et impressions organisationnelles devient alors un enjeu stratégique pour les chefs d'établissement et leurs équipes. Tirées de recherches menées dans quatre pays (Belgique, France, Angleterre, Chili) aux réalités contrastées, les douze contributions rassemblées montrent que l'école contemporaine est soumise à une double tendance de standardisation et de différentiation.

DUPRIEZ Vincent, DUMAY Xavier, "Les quasi-marchés scolaires : au bénéfice de qui ?", Revue française de pédagogie, $n^{\circ}$ 176, septembre 2011, p. 83-100, bibliogr.

La figure du marché a depuis quelques décennies fait irruption dans le champ scolaire et est présentée par certains auteurs comme une forme de coordination alternative à la régulation par les pouvoirs publics, susceptible de mieux rencontrer les objectifs poursuivis par les systèmes éducatifs. À travers une analyse secondaire de la base de données PISA 2006, les auteurs mettent à l'épreuve cette hypothèse, ainsi qu'une hypothèse alternative qui suggère que le quasi-marché, davantage que d'augmenter le niveau moyen d'efficacité des systèmes scolaires, accentue la ségrégation et ses effets sur les compétences des élèves.

FELOUZIS Georges, MAROY Christian, VAN ZANTEN Agnès, Les marchés scolaires : sociologie d'une politique publique d'éducation, PUF/Paris, 2013, $217 p$.

Les marchés scolaires constituent un mode d'organisation de l'école dans de nombreux pays. Que faut-il comprendre par "marchés scolaires » ? Comment fonctionnent-ils ? Quel est leur impact sur l'efficacité et l'équité de l'école? Les auteurs s'appuient sur des recherches internationales pour analyser les politiques de marché scolaire dans plusieurs pays. Après avoir donné une définition des marchés scolaires, ils en dressent une typologie : les marchés privés, les quasi-marchés et les marchés officieux. 


\title{
DÉVELOPPEMENT DE L'ENSEIGNEMENT PRIVÉ
}

\author{
BOESKENS Luka, Regulating publicly funded private schools: a literature review \\ on equity and effectiveness, OCDE/Paris, novembre 2016, 67 p. [en ligne]
}

Le libre choix d'une école devient une caractéristique de plus en plus commune des systèmes éducatifs des pays de l'OCDE. La réglementation des écoles privées subventionnées par l'État est devenue une préoccupation saillante pour les chercheurs et les décideurs. En se concentrant sur trois domaines de la réglementation - l'admission sélective, les frais de scolarité et les établissements à but lucratif - ce rapport présente une revue de la littérature des effets du libre choix sur l'équité et l'efficacité pédagogique. L'admission sélective et les frais de scolarité sont susceptibles d'aggraver la ségrégation sociale et de réduire les incitations faites aux écoles de concourir sur la base de leur qualité éducative. [http://bit-ly/2BB8svd]

DAHAL Mahesh, NGUYEN Quynh, Private non-state sector engagement in the provision of educational services at the primary and secondary levels in South Asia: an analytical review of its role in school enrollment and student achievement, Banque mondiale/Washington, juin 2014, 32 p.

L'engagement du secteur privé (non étatique) dans la prestation de services éducatifs aux niveaux primaire et secondaire en Asie du Sud a récemment connu une croissance importante. Ce type d'éducation regroupe différentes formes : les écoles financées et gérées par le secteur privé, les écoles financées par le gouvernement et gérées par le secteur privé, des bons pour les écoles privées et des tutorats organisés en dehors de la classe. Toutefois, les résultats des élèves varient considérablement entre les établissements de chaque type, avec de nombreuses écoles privées aux résultats faibles ainsi que des écoles publiques aux bons résultats. [http://goo.glaFKvEQ]

DUTERCQ Yves, Où va l'éducation entre public et privé ?, De Boeck/Bruxelles, 2011, $202 p$.

L'opposition entre public et privé a longtemps structuré la manière de voir et d'interpréter l'action dans le domaine de l'éducation. Or il existe une grande diversité de formes d'enseignement privé dans le monde, à la mesure des histoires nationales en matière d'éducation, des traditions politiques et des situations économiques. Il importe de distinguer entre enseignement privé et mouvement de privatisation de l'enseignement. L'ouvrage propose une analyse de l'expansion de la privatisation et de la marchandisation de l'éducation à partir de l'étude de cas diversifiés, issus de différents espaces politiques: Angleterre, Argentine, Belgique francophone, Chili, France, Israël, Québec, Suisse.

\section{MACPHERSON Ian, ROBERTSON Susan, WALFORD Geoffrey (ed.), Education, privatisation and social justice: case studies from Africa South Asia and South East Asia, Symposium Books/Oxford, 2014, 310 p.}

L'implication d'acteurs privés dans l'éducation n'est pas nouvelle mais des points critiques sont apparus durant cette décennie. Ce volume explore les formes émergentes du secteur privé dans l'éducation au travers d'études en Afrique (Kenya, Ghana, Nigéria) et en Asie (Bangladesh, Cambodge, Népal, Pakistan, Philippines, Vietnam). À la lumière de ces analyses, ce phénomène de privatisation existe à tous les niveaux des systèmes éducatifs, de l'appareil administratif à l'élaboration des politiques, de l'accès aux structures d'éducation formelle jusqu'aux activités extra-scolaires. Par ailleurs, l'éducation est un secteur qui s'ouvre de manière croissante aux entreprises privées et aux intérêts commerciaux. 
MOUMNE Rolla, SAUDEMONT Charlotte, Overview of the role of private providers in education in light of the existing international legal framework: investments in private education: undermining or contributing to the full development of the human right to education? UNESCO/Paris, 2015, 32 p. [en ligne]

Ce document vise à donner une vue d'ensemble du rôle des prestataires privés d'éducation au regard du droit à l'éducation et du cadre juridique international. Une première partie rappelle brièvement la nature des obligations qui incombent aux États pour la mise en ouvre du droit à l'éducation. Les trois parties suivantes présentent les différents types d'enseignement privé, ainsi que les nouveaux défis liés à la privatisation et à la marchandisation de l'éducation, soulignant les impacts, positifs et négatifs du phénomène. [https://goo.g//8aZuC6]

\title{
RIZVI Fazal, Privatisation de l'éducation : tendances et conséquences, UNESCO/
} Paris, octobre 2016, 13 p.

La privatisation de l'éducation a les faveurs de nombreux gouvernements, et a souvent été soutenue par des organisations intergouvernementales, des entreprises transnationales et des organisations non gouvernementales. Cette étude donne un aperçu des débats sur la privatisation de l'éducation et en analyse les effets sur les inégalités sociales et la distribution inéquitable des chances en matière d'éducation. Elle conclut que, puisque l'éducation ne peut plus être entièrement financée et assurée par l'État, la question est moins de savoir si l'engagement des acteurs privés dans le secteur de l'éducation est ou non une bonne chose que de déterminer dans quelle mesure, comment et à quelle fin l'État devrait réglementer leurs activités.

[https://goo.gl/ksNBUL]

\section{LA CONCURRENCE ENTRE ÉTABLISSEMENTS SCOLAIRES}

\author{
ANDREWS Jon, HUTCHINSON Jo, JOHNES Rebecca, Grammar schools and \\ social mobility, London: Education Policy Institute, 2016, 51 p. [en ligne]
}

Devant la décision du gouvernement anglais d'encourager le développement des Grammar schools, écoles secondaires sélectives, l'Education Policy Institute a étudié l'impact de la sélection dans ces établissements scolaires sur les résultats des élèves et la mobilité sociale en Angleterre, en utilisant les données des tableaux des résultats des écoles, du recensement scolaire et de la base de données nationale des élèves. Il considère qu'au niveau national, un plus grand nombre de ces écoles secondaires risquerait d'entraîner de faibles gains pour la minorité d'enfants fréquentant ces écoles, y compris le petit nombre d'enfants issus de milieux défavorisés. [https://goo.g/p5UaSg]

BROCCOLICHI Sylvain, CHOUKRI Ben-Ayed, TRANCART Danièle (coord.), École: les pièges de la concurrence. Comprendre le déclin de l'école française, Paris : La Découverte, 2010, 312 p.

En quoi le développement des concurrences et des disparités entre établissements influe-t-il sur l'inégale réussite des élèves? L'ouvrage prend appui sur une recherche de grande ampleur consacrée aux disparités territoriales d'éducation en France, avec l'objectif de rendre compte de la variabilité de la réussite scolaire des élèves en fonction du lieu de scolarisation. L'ouvrage s'achève sur l'analyse des évolutions très négatives de nombreux territoires urbains, en montrant en quoi l'essor de la concurrence et le manque de régulation politique ont contribué au déclin de l'école française. 
COMBAZ Gilles, "Concurrence entre établissements et choix de l'école : les personnels de direction mis à l'épreuve? ", Éducation et sociétés, 2013, $n^{\circ} 31$, 2013/1, p. 157-170

Parmi les transformations récentes qui ont affecté le fonctionnement des systèmes éducatifs de la plupart des pays occidentaux, le choix de l'école par les familles et la concurrence entre les établissements représentent deux dimensions importantes. En France, des mesures ont été prises en 2007 pour étendre la liberté de choix des familles. Ceci a contribué indirectement à mettre en concurrence les établissements par le biais des caractéristiques sociales des élèves qu'ils étaient susceptibles d'attirer. Les personnels de direction sont directement concernés par ces phénomènes. [https://g00.g/pYhvgD]

DUMAY Xavier, DUPRIEZ Vincent, MAROY Christian, " La concurrence entre écoles accroît-elle les effets de composition? ", Éducation comparée, 2011, vol. 6, p. 49-68, bibliogr.

La ségrégation entre écoles se caractérise par des différences dans la composition des établissements. Les auteurs de cet article s'intéressent en particulier aux effets de composition, c'est-à-dire à l'influence sur chacun des élèves des caractéristiques globales de l'ensemble des élèves de leur établissement. Ils y proposent un schème d'interprétation qui a pour but de raisonner sur les conditions institutionnelles favorables à l'émergence des effets de composition. Ce schème vise à rendre compte de la diversité des résultats de recherches relatives aux effets de composition, en posant l'hypothèse que ceux-ci sont maximisés dans un environnement institutionnel qui combine une latitude dans le choix de l'école et une autonomie importante des établissements.

HUGON Marie-Anne, LAWES Shirley, "Innovation ou fragmentation d'un système public d'enseignement? L'ouverture des Free Schools en Angleterre », Spécificités, 2017/1, $n^{\circ}$ 10, p. 201-217

Les Free Schools anglaises résultent de la politique scolaire menée par le gouvernement britannique depuis 2010. Tout en étant financées par le ministère de l'éducation, elles peuvent être montées par des parents, des enseignants, des associations et tout groupe désireux de répondre à une demande scolaire locale identifiée. En prenant appui sur trois études de cas, les auteures présentent l'état du débat autour de ces établissements.

FRANKENBERG Erica, KOTOK Stephen, SCHAFFT Kai, « Exploring school choice and the consequences for student racial segregation within Pennsylvania's charter school transfers ", Education policy analysis archives, mars 2017, vol. 25, $n^{\circ} 22$ [en ligne]

Cette étude explore dans quelle mesure la composition raciale des "écoles à charte " (charter schools) peut être un facteur important dans les choix scolaires des élèves ségrégués. Les résultats indiquent que les élèves noirs et latino-américains sont fortement opposés à aller dans des écoles à charte avec des pourcentages plus élevés d'élèves blancs. Inversement, les élèves blancs sont plus susceptibles de s'inscrire dans ces établissements. À mesure qu'augmente le pourcentage et le nombre d'élèves transférés dans les écoles à charte, les choix d'auto-ségrégation soulèvent des questions critiques concernant l'équité en matière d'éducation et les effets des réformes éducatives et des politiques scolaires sur la promotion d'environnements éducatifs diversifiés. [http://dx.doi.org/10.14507/epaa.25.2601]

\section{HURTEAU Philippe, DUCLOS Anne-Marie? Inégalité scolaire : le Québec dernier} de classe ? IRIS : Institut de recherche et d'informations socioéconomiques/Montréal, septembre 2017, 12 p. [en ligne]

La concurrence entre les différents établissements scolaires (le "marché scolaire ») s'impose comme la nouvelle norme présidant au destin des écoles québécoises. Que ce soit par le biais de la concurrence faite par les écoles privées aux écoles publiques ou même, de manière plus 
insidieuse, de la chasse à la "clientèle » qui oppose les écoles publiques les unes aux autres, certains semblent penser qu'une configuration concurrentielle du réseau d'éducation l'aiderait à mieux répondre aux aspirations des élèves. Pourtant, cette mise en concurrence ne fait que miner la qualité des services offerts à la majorité des jeunes. [http://bit.ly/2vPAtlj]

\section{SÉGRÉGATION SCOLAIRE ET COMMUNAUTARISME}

\section{MERLE Pierre, La ségrégation scolaire, La Découverte/Paris, 2012, 126 p.}

La ségrégation scolaire en France renouvelle, progressivement et profondément, les modalités de scolarisation des élèves et accentue les inégalités. Depuis la fin des années 1970, les transformations économiques et idéologiques (chômage structurel, déclassement des jeunes diplômés, individualisme croissant, etc.) ont débouché sur de nouvelles politiques éducatives. L'auteur définit dans une première partie la "ségrégation scolaire » qui revêt quatre dimensions : le sexe, l'origine ethnique, les compétences scolaires et l'origine sociale. L'auteur s'appuie sur les comparaisons internationales pour montrer que le niveau de "ségrégation scolaire " varie considérablement selon les organisations éducatives nationales.

OCDE, «Concurrence entre les établissements d'enseignement : quand est-elle bénéfique? ", PISA à la loupe, août 2014, 4 p. [en ligne]

Dans la plupart des systèmes d'éducation, plus de $50 \%$ des élèves de 15 ans fréquentent un établissement en concurrence avec un autre pour attirer les élèves d'une même zone résidentielle. Parmi les pays et économies à l'étude, la performance ne présente pas de lien avec le fait que les établissements soient en concurrence ou non pour leurs effectifs d'élèves. Dans les systèmes où les parents ont la possibilité de choisir l'établissement de leur enfant et où les établissements sont en concurrence pour leurs effectifs d'élèves, il existe souvent une forme plus marquée de ségrégation sociale entre les établissements. [http://dx.doi.org/10.1787/5jz15937b3mv-fr]

MINCU Monica E., "School community parent participation and non-neutrality: globalisation challenges and communitarian responses ", Spirale : revue de recherches en éducation, 2013, $n^{\circ} 51, p .141-153$

Le communautarisme s'est développé en réponse à un individualisme croissant et il a des conséquences majeures dans le domaine des politiques de l'éducation et de leur théorisation. Au moment où des approches tendent à durcir les tensions entre processus de mondialisation et identités cosmopolites, certaines perspectives soulignent combien la reconnaissance des identités culturelles et religieuses dans l'espace public constitue un mouvement bien inscrit dans la mondialisation. Dans cet article, l'auteure questionne les enjeux liés à la non-neutralité en éducation en tant que modèle pertinent et dont la diffusion croissante s'enracine dans l'idéal communautaire. L'auteure s'intéresse à la thèse d'une éducation libérale « réformée » et explore notamment les perspectives théoriques de la non-neutralité.

PONS Xavier, ROBINE Florence (coordinateurs), "Les attentes éducatives des familles : dossier », Revue internationale d'éducation de Sèvres, avril 2013, $n^{\circ}$ 62, p. 31-155 [en ligne]

Les attentes éducatives des familles constituent un point nodal particulièrement riche pour l'étude de nombreux phénomènes éducatifs et ce, dans divers contextes socio-culturels. Quatre tendances se dégagent des dix études proposées dans ce numéro : l'intensification des attentes concerne toutes les familles dans de nombreux pays ; ces attentes varient fortement selon des groupes sociaux ; elles sont façonnées par les transformations des systèmes scolaires et des rôles endossés par l'École; cette élévation des attentes s'accompagne souvent d'une défiance croissante vis-à-vis de l'École. [https://ries.revues.org/3059] 
OCDE : Organisation de coopération et de développement économiques, School choice and school vouchers: an OECD perspective, OCDE/Paris, mai 2017, 27 p. [en ligne]

Au cours des dernières décennies, plusieurs pays de l'OCDE ont adopté des politiques facilitant le choix d'une école par les parents. Le système des chèques éducation (school vouchers) permet aux familles de financer directement l'école où leur enfant sera scolarisé. Il suscite de nombreux débats. Les partisans du libre choix de l'école défendent le droit des parents et les effets stimulants de la concurrence entre les établissements scolaires, qui inciterait à innover et à améliorer la qualité de l'enseignement. Les critiques de ce système estiment que les élèves des milieux favorisés choisissent de préférence l'enseignement privé, que ce système génère une ségrégation sociale accrue et que les ressources publiques sont détournées au profit de l'enseignement privé. S'appuyant sur les données de l'OCDE et une analyse de la situation dans des pays où ce système a été introduit (Belgique néerlandophone, Chili, Danemark, Estonie, Danemark, Pays-Bas), ce document examine ces questions. [http://bit.ly/2rcFdN7]

\section{WEST Anne, HIND Audrey, Secondary school admissions in London 2001 to} 2015: compliance complexity and control, London: LSE Academic publishing, mars 2016, 26 p. [en ligne]

Ce rapport de recherche présente une analyse des critères et pratiques d'admission des établissements d'enseignement secondaire de Londres entre 2001 et 2015. La proportion d'écoles secondaires responsables des admissions de leurs élèves est passée d'un peu plus de $40 \%$ en 2001 à près de $80 \%$ en 2015. Les conditions d'admission doivent être claires, équitables et objectives. Cependant, des écoles responsables de leurs propres admissions (écoles à caractère religieux, academies) ont de nombreux et complexes critères de sélection.

[https://goo.gl/gTzE9w]

WIBORG Susanne, LARSEN Kristina R., "Why school choice reforms in Denmark fail: the blocking power of the teacher union? ", European journal of education, 2017, vol. 52, $n^{\circ}$ 1, p. 92-103, [en ligne]

Au Danemark, les différents gouvernements ont initié depuis vingt ans des réformes favorisant le choix d'un établissement scolaire par les parents. Cependant, seul $12 \%$ des parents choisissent un établissement scolaire public différent de celui qui est attribué à leur enfant. Cet article examine pourquoi le choix d'un établissement scolaire est exercé de manière limitée par les parents malgré d'importantes initiatives visant à accroître la concurrence entre les établissements scolaires ainsi que les possibilités de choix. Cela s'explique principalement par une similarité relativement élevée des établissements scolaires, due à un consensus entre les municipalités et le syndicat des enseignants. [https://goo.gl/3YYTQf]

\section{RÉSEAUX ÉDUCATIFS INTERNATIONAUX}

ANGEY-SENTUC Gabrielle, "Le mouvement musulman turc de Fethullah Gülen en Afrique subsaharienne: faire l'école au transnational ", Politique africaine, 2015, vol. 139, $n^{\circ} 3$, p. 23-42

Cet article porte sur l'insertion d'un acteur religieux transnational originaire de Turquie dans les champs éducatifs d'Afrique subsaharienne. Le mouvement musulman de Fethullah Gülen est composé d'hommes d'affaires, d'intellectuels et d'enseignants turcs qui centrent leur action sur l'ouverture d'écoles dans le monde. L'insertion de ces écoles en Afrique subsaharienne, à partir de la deuxième moitié des années 1990, s'intègre dans le cadre d'une libéralisation des secteurs éducatifs du sous-continent. 
BUNNELL Tristan, FERLIG Michael, JAMES Chris, "What Is International about International Schools? An Institutional Legitimacy Perspective », Oxford review of education, 2016, vol. 42, $n^{\circ} 4$, p. 408-423

Ces dernières années, le nombre d'écoles internationales dans le monde a augmenté rapidement en grande partie grâce à la croissance de nouvelles formes d'écoles internationales, qui diffèrent nettement des formes traditionnelles. Ces nouvelles formes sont souvent exploitées à des fins commerciales ; elles sont généralement destinées aux enfants de la population locale (indigène) riche et ont été définies comme des écoles internationales parce qu'elles sont situées dans un pays non anglophone et que l'anglais est le moyen de communication de l'école.

\section{HAYDEN Mary, THOMPSON Jeff (ed.), International schools: current issues and} future prospects, Symposium Books/Oxford, 2016, vol. 26, $n^{\circ}$ 2, 240 p., bibliogr.

Les premières écoles internationales datent d'il y a plus d'un siècle et leur croissance est fulgurante, ces dernières années. Leur nombre a augmenté, elles se sont répandues dans différentes régions du monde, les types d'établissements se sont diversifiés ainsi que les modes de propriété. Aussi, de nombreuses questions complexes se posent quant à leurs objectifs fondamentaux, aux programmes qu'elles choisissent, à la nature de leur organisation et à leurs contributions potentielles pour répondre à un besoin éducatif mondial. Quelle influence ces écoles internationales ont-elles sur les systèmes scolaires nationaux?

\section{ÉCOLES ALTERNATIVES OU FORMES ALTERNATIVES D'ENSEIGNEMENT}

DIA Hamidou, HUGON Clotilde, D'AIGLEPIERRE Rohen (coordinateurs), "États réformateurs et éducation arabo-islamique en Afrique : dossier ", Afrique contemporaine, 2016, $n^{\circ} 257$, p. 3-110

Alors qu'elle est restée longtemps en marge des politiques nationales en Afrique, l'éducation arabo-islamique constitue une offre éducative en fort développement. Ce dossier dresse un panorama de l'offre arabo-islamique, sous l'angle des institutions, des acteurs, des systèmes de financement et des modes de gouvernance. Il passe en revue les savoirs et les philosophies religieuses qui les sous-tendent et analyse les réformes publiques destinées à rapprocher l'offre éducative arabo-islamique des standards scolaires classiques.

HUGON Marie-Anne, VIAUD Marie-Laure (coordinatrices), "Les écoles et pédagogies "différentes" : approches internationales ", La revue française d'éducation comparée, Paris : L'Harmattan, mai 2017, $n^{\circ} 15,206$ p.

Ce numéro est consacré aux pédagogies et/ou écoles différentes dans le monde, c'est-à-dire aux classes et aux écoles qui, à tous les niveaux scolaires, sont repérées dans le paysage éducatif comme alternatives, expérimentales et/ou se réclamant du courant des pédagogies nouvelles. L'ensemble des articles vise à donner un premier aperçu de l'état des écoles différentes aujourd'hui dans le monde.

\section{MOLNAR Alex (ed.), Virtual schools in the U.S 2017, National education policy} center/Boulder, avril 2017, 103 p., [en ligne]

Ce rapport annuel ( $5^{\mathrm{e}}$ édition) offre un aperçu détaillé des écoles virtuelles à temps plein et des écoles hybrides (combinaison d'enseignement traditionnel et d'enseignement virtuel) aux États-Unis. Dans une première partie, le rapport recense ces écoles et fournit une analyse démographique des élèves, qui diffèrent des élèves des écoles publiques traditionnelles et ne réussissent pas aussi bien que dans celles-ci. Néanmoins, la croissance des inscriptions se 
poursuit. Les recherches menées sur l'efficacité de ce type d'écoles, de la maternelle à la $12^{\mathrm{e}}$ année, montrent que les élèves ont des niveaux bien inférieurs à ceux des écoles traditionnelles. [https://goo.glu7ig2W]

\section{REDFORD Jeremy, BATTLE Danielle, BIELICK Stacey, Homeschooling in the} United States: 2012, NCES : National center for education statistics, novembre 2016, 52 p.

Ce rapport fournit des estimations du nombre d'élèves scolarisés à la maison aux États-Unis en 2012 et compare ces estimations avec celles de 1999, 2003 et 2007. Il analyse les caractéristiques sociodémographiques de ces élèves ainsi que les raisons de ce choix par les parents et les ressources en ligne utilisées par les familles. Le pourcentage d'élèves âgés de 5 à 17 ans scolarisés à domicile a progressé, passant de $1,7 \%$ en 1999 à 3,4 \% en 2012 , soit 1,8 million d'élèves. Environ un tiers des parents d'élèves scolarisés à domicile ont suivi un cours de préparation. [http://bit.ly/2iajuVW]

\section{ROTHERMEL Paula ed., International perspectives on home education: do we} still need schools, Palgrave Macmillan/Basingstoke, 2015, 333 p.

L'ouvrage présente des situations très contrastées de l'éducation à domicile dans le monde (Royaume-Uni, États-Unis, Australie, Israël, Afghanistan, Norvège, Allemagne). Les contributions sont organisées selon différents angles d'analyse : processus d'apprentissage, tensions et critiques, conflit politique, mode de vie et choix, modèles de formation à domicile ou relations culturelles et interculturelles. 